\title{
CoolCalc: A Long-Haul Truck Thermal Load Estimation Tool
}

\author{
Jason A. Lustbader, John P. Rugh, Brianna R. Rister and Travis S. Venson \\ National Renewable Energy Laboratory
}

\begin{abstract}
In the United States, intercity long-haul trucks idle approximately $1,800 \mathrm{hrs}$ per year primarily for sleeper cab hotel loads, consuming 838 million gallons of diesel fuel [1]. The U.S. Department of Energy's National Renewable Energy Laboratory (NREL) is working on solutions to this challenge through the CoolCab project. The objective of the CoolCab project is to work closely with industry to design efficient thermal management systems for long-haul trucks that keep the cab comfortable with minimized engine idling. Truck engine idling is primarily done to heat or cool the cab/ sleeper, keep the fuel warm in cold weather, and keep the engine warm for cold temperature startup. Reducing the thermal load on the cab/sleeper will decrease air conditioning system requirements, improve efficiency, and help reduce fuel use. To help assess and improve idle reduction solutions, the CoolCalc software tool was developed. CoolCalc is an easy-to-use, simplified, physics-based heating, ventilating and air conditioning (HVAC) load estimation tool that requires no meshing, has flexible geometry, excludes unnecessary detail, and is less time-intensive than more detailed computer-aided engineering (CAE) modeling approaches. It is intended for rapid trade-off studies, technology impact estimation, and preliminary HVAC sizing design. It also complements more detailed and expensive CAE tools by exploring and identifying regions of interest in the design space. CoolCalc is built on NREL's OpenStudio platform and is a plug-in extension of Google's SketchUp software. This paper describes the CoolCalc tool, provides outdoor long-haul truck thermal testing results, shows validation using these test results, and discusses future applications of the tool.
\end{abstract}

\section{INTRODUCTION}

Heating and air conditioning are two of the primary reasons for long-haul truck main engine operation when the vehicle is parked. In the United States, trucks that travel more than 500 miles per day use 838 million gallons of fuel annually for overnight idling [1]. Including workday idling, over 2 billion gallons of fuel are used annually for truck idling [2]. By reducing thermal loads and improving efficiency, there is a great opportunity to reduce the fuel used and emissions created by idling. Reducing the thermal load for truck cab/ sleepers will enable cost-effective idle reduction solutions. If the fuel savings from new technologies can provide a 3- to 5year payback time, fleet owners will be economically motivated to incorporate them. This provides a pathway to rapid adoption of effective thermal load reduction solutions.

The U.S. Department of Energy's (DOE's) National Renewable Energy Laboratory (NREL) CoolCab project is researching efficient thermal management systems that keep the cab occupants comfortable without the need for engine idling. The CoolCab research approach is to reduce thermal loads, concentrate on occupant thermal comfort, and maximize equipment efficiency. By working with industry partners to develop and apply commercially viable solutions that reduce idling fuel use, both national energy security and sustainability will be improved. To achieve this goal, NREL is developing tools and test methods to assess idle reduction technologies. The truck cab industry needs a high-level analysis tool to predict thermal loads that are used to evaluate load reduction technologies and their impact on climatecontrol fuel use.

To meet this need, NREL has developed CoolCalc, a software tool to assist the industry in reducing climate control loads for heavy-duty vehicles. CoolCalc is a heating, ventilating and air conditioning (HVAC) load estimation tool 
that enables rapid exploration of idle reduction design options for a range of climates.

\section{APPROACH}

\section{COOLCALC MODEL DEVELOPMENT}

CoolCalc is an easy-to-use, simplified, physics-based HVAC load estimation software tool that requires no meshing, has flexible geometry, excludes unnecessary detail, and is less time-intensive than more detailed computer-aided engineering (CAE) modeling approaches. It is intended for rapid trade-off studies, technology impact estimation, and preliminary HVAC sizing design. It complements more detailed and expensive CAE tools.

CoolCalc is built on NREL's OpenStudio platform. This was done to accelerate development and leverage previous and ongoing DOE investments. OpenStudio was developed at NREL and released in 2008. It is a plug-in extension of Google's SketchUp software. DOE's EnergyPlus is used as the heat transfer solver. EnergyPlus is a DOE-funded software package designed for building efficiency analysis, which was found to be general enough to extend to truck cab thermal modeling.

Unlike previous building thermal simulation programs, EnergyPlus provides a fully integrated simulation where the building HVAC zones, system, and plant (source) are solved together. This provides a more physically realistic solution and allows for more detailed control implementation. Heat transfer is described by a set of time-dependent energy and moisture balances, and the resulting ordinary differential equations are solved using a predictor-corrector approach. For solar loading, an anisotropic radiance model is used, allowing the superposition of three components: isotropic radiance, point source circumsolar brightening at the sun, and horizon brightening. The sun position is tracked as a function of geographic location, time of year, and time of day. A shading model is implemented that accounts for shadowing of surfaces by other surfaces. EnergyPlus's window model is based on Lawrence Berkeley National Laboratory's WINDOW program algorithms and uses solar transmittance, reflectance, and absorptance properties. Full spectral analysis is also possible. All surfaces are treated as gray bodies. The external surface heat transfer balance includes shortwave solar radiation, longwave thermal radiation, convection, and conduction through the walls. Conduction is one dimensional through the thickness of a wall. Walls are defined using a series stack-up of materials that allow for thermal storage. The interior surface heat transfer modes include shortwave solar radiation from windows, longwave radiation, and convection. The internal radiation view factors are approximated by a ratio of "seen" areas, then corrected for reciprocity and completeness. A detailed description of EnergyPlus's modeling and solution methods is beyond the scope of this paper; for further details see the extensive documentation available [ $\underline{3}]$.

While CoolCalc is flexible and does not dictate a specific process, a typical workflow (illustrated in Figure 1) might begin with the creation of geometry using the Parametric $\mathrm{Cab}$ creation tool (Figure 2). The Parametric $\mathrm{Cab}$ creation window has a series of tabs across the top, one for each air zone in the model. Each tab has a list of available parametric variables that will modify the geometry. These variables and the parametric geometry relationships are determined by a model definition file created in the geometry coding framework. The user can also switch between the available parametric cab models to find one that that best suits their needs. To the right of the units, the allowable variable range is displayed. To illustrate this parametric capability, the windscreen angle was changed from $60^{\circ}$ to $80^{\circ}$ (Figure 3 ). The cab model quickly updates, allowing for fast modification of the geometry.

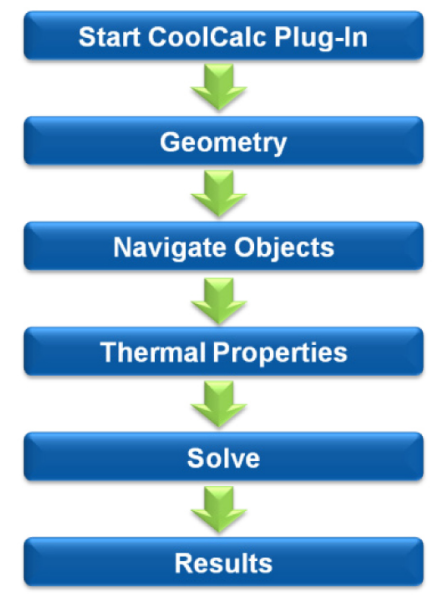

Figure 1. Typical CoolCalc workflow

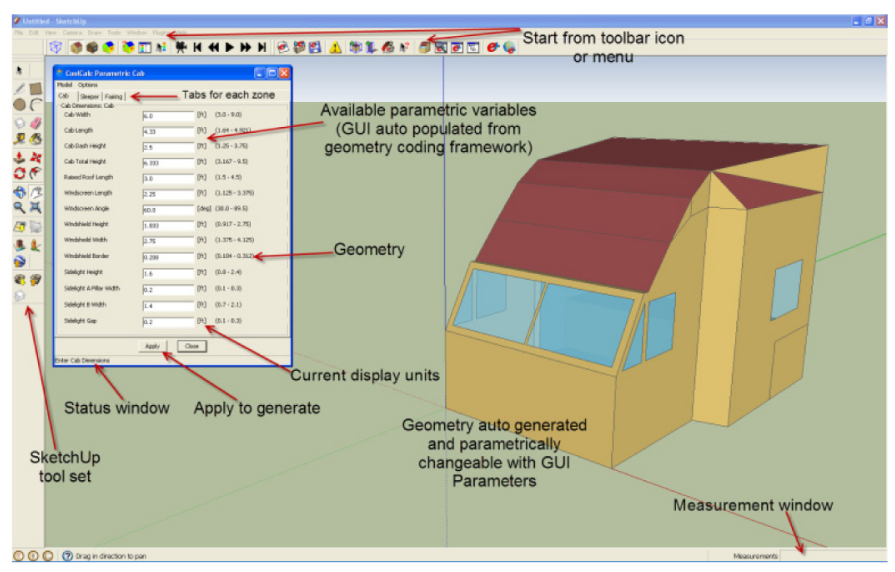

Figure 2. Parametric Cab geometry creation 


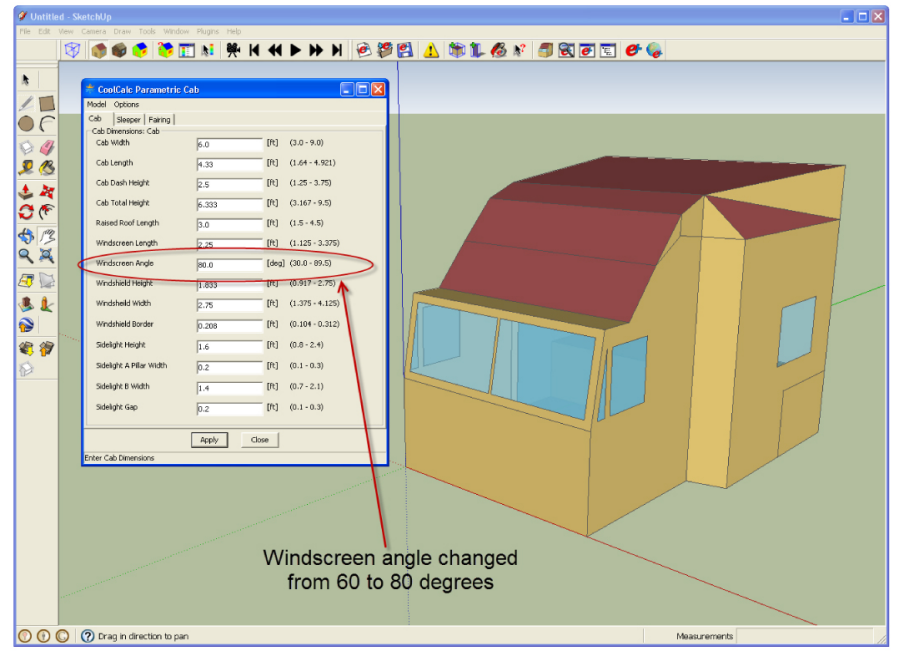

Figure 3. Parametric Cab with modified windscreen angle

Once the cab geometry is established using the Parametric $\mathrm{Cab}$ tool, it can be manually modified by the user. Figure 4 shows an example of a user adding an additional sidelight to the sleeper cab. The sleeper cab sidewall was activated by double clicking on the surface. Once activated, the SketchUp drawing tools can be used to modify the geometry. The dashed lines are construction lines that were created to help quickly draw the sidelight and can be easily hidden or deleted later. The pencil tool was then used to trace out the construction lines. The pencil tooltip icon can be seen in the top left corner of the sidelight. Once the window shape is closed by the pencil tool, it is automatically recognized as a window and assigned default properties.

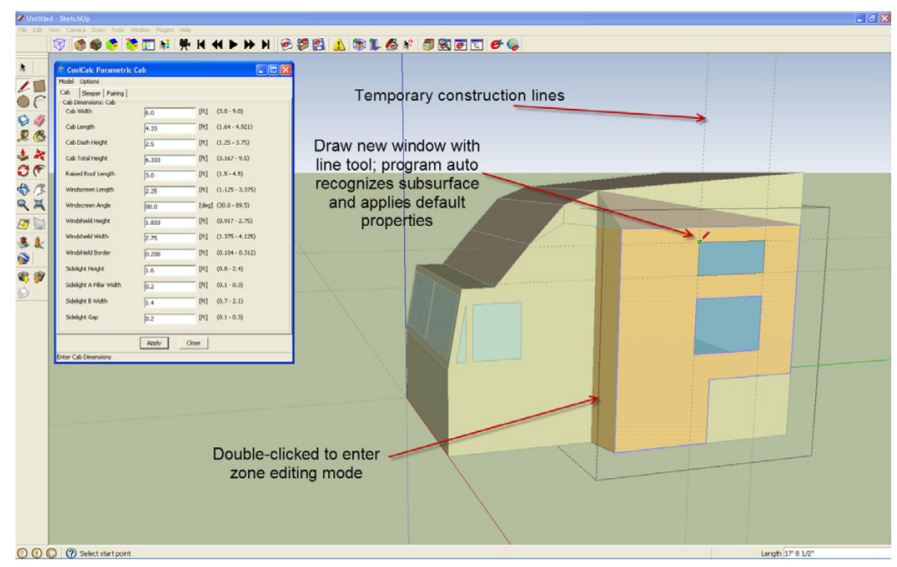

Figure 4. Manual modification of geometry: adding a sidelight

In EnergyPlus, every component of the model, e.g., walls, materials, location, and solver time step, is treated as an object. In CoolCalc, to modify or define new objects the Object Browser tool is opened (Figure 5). On the left side of the Object Browser window is the object tree, which shows all the objects that are available in the model and allows creation of new objects. Below the object tree is the library window. The library window allows the user to load and manipulate additional libraries of objects. These objects can then be added to the current model by dragging and dropping them into the object tree. Below the specific object window interface, to the right of the object tree, is the text editing window. This window allows for manual modification of the current object, giving full control to advanced users. For objects where no specific interface has been developed, the text editing window will comprise the entire right side of the split window. All object windows also provide a "Comment" option in the upper right corner to help users document their object assumptions.

To modify or define new materials, a material object is selected in the object tree (Figure 5). Based on this object tree selection, the material definition window is displayed to the right of the object tree. The material definition window provides text boxes or pulldown menus for all the basic material thermal properties: name, roughness, thickness, conductivity, density, specific heat, and radiation absorptance.

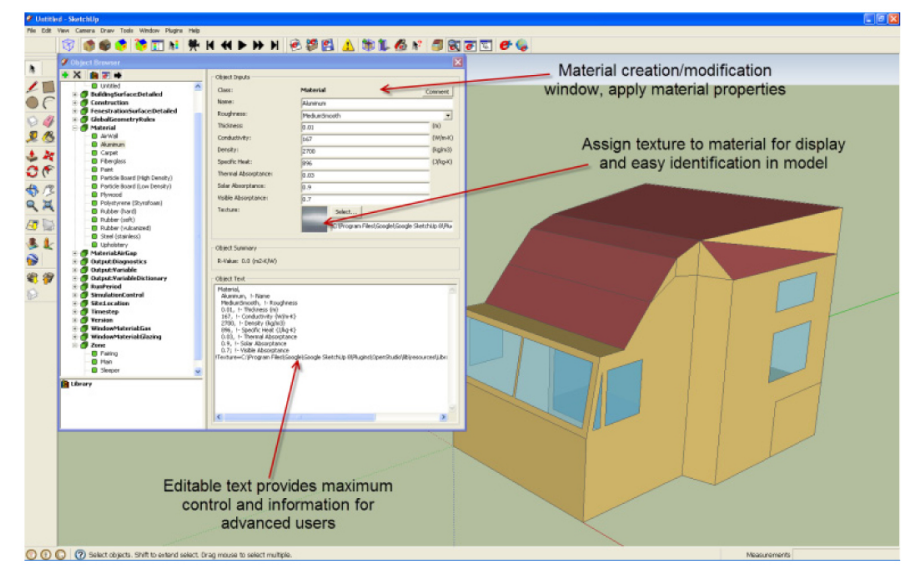

Figure 5. Object Browser and Material Definition Window

Each surface in CoolCalc is treated as multiple layer, onedimensional conduction, forming a "sandwich" type structure. To define this layered structure, a construction object is used. Once again navigating the object tree, a construction object is selected. This changes the current object window (right side) to display the construction definition window (Figure 6). In this window, the user selects the materials to include in the construction and can change their order. Materials assigned to the inner and outer layers will determine the solar radiation properties and the texture displayed in the texture-rendering mode. 


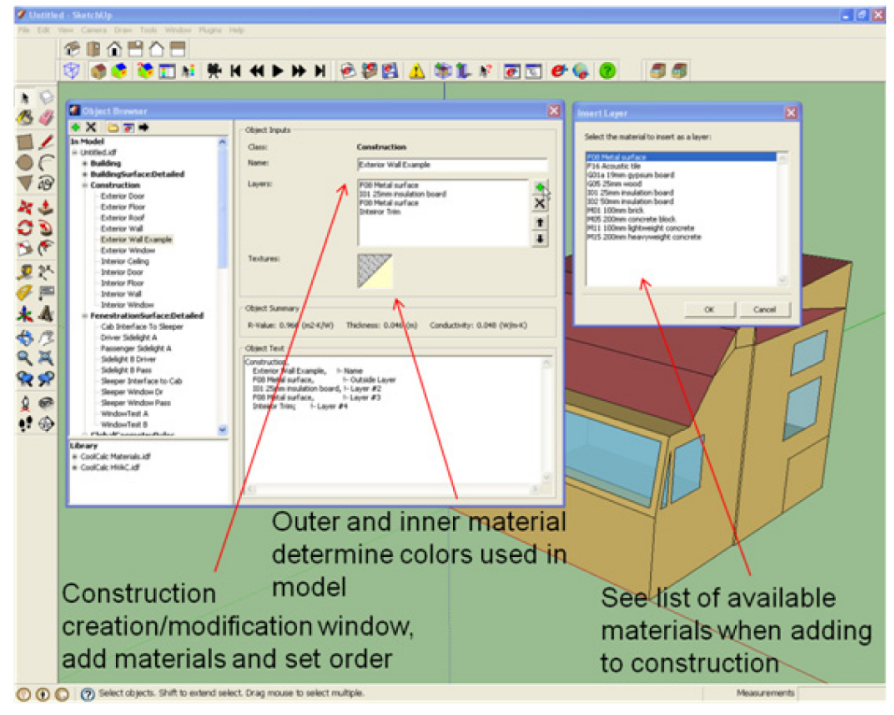

Figure 6. Construction Definition Window

Figure 7 shows the model in construction rendering mode. In this mode, the inner and outer surfaces are colored by their respective construction's material textures. The window to the left of the cab is the Construction Palette. It allows sorting and selection of constructions and their application to the model using a point-and-click paint can tooltip.

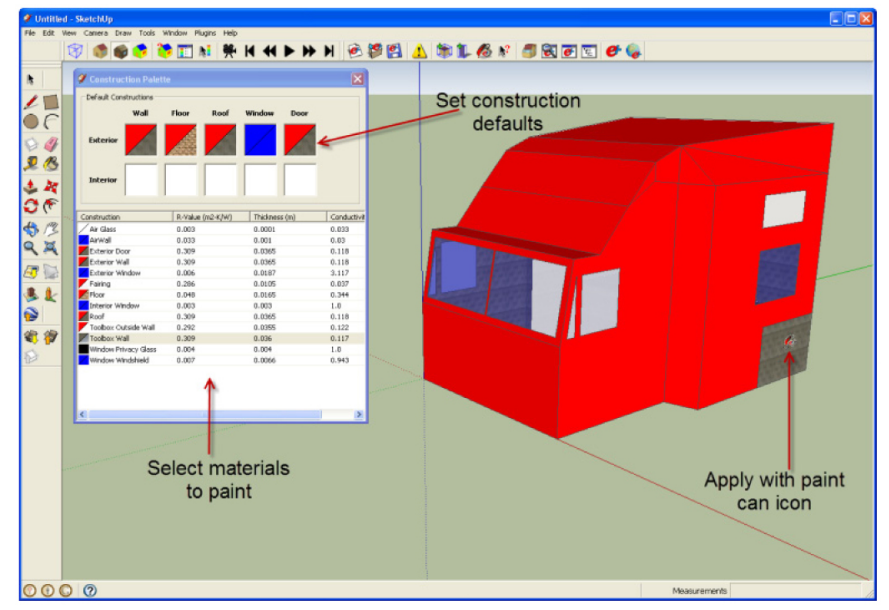

Figure 7. Construction palette window and texture rendering mode

Before solving the model, a weather file is selected. There are currently Typical Mean Year data available for 2,100 locations worldwide. Custom weather data can also be entered. A simulation period of one day to one year is also selected. Once the model is solved, the results can be displayed within the interface. Figure 8 shows the exterior temperatures displayed on the truck cab/sleeper.

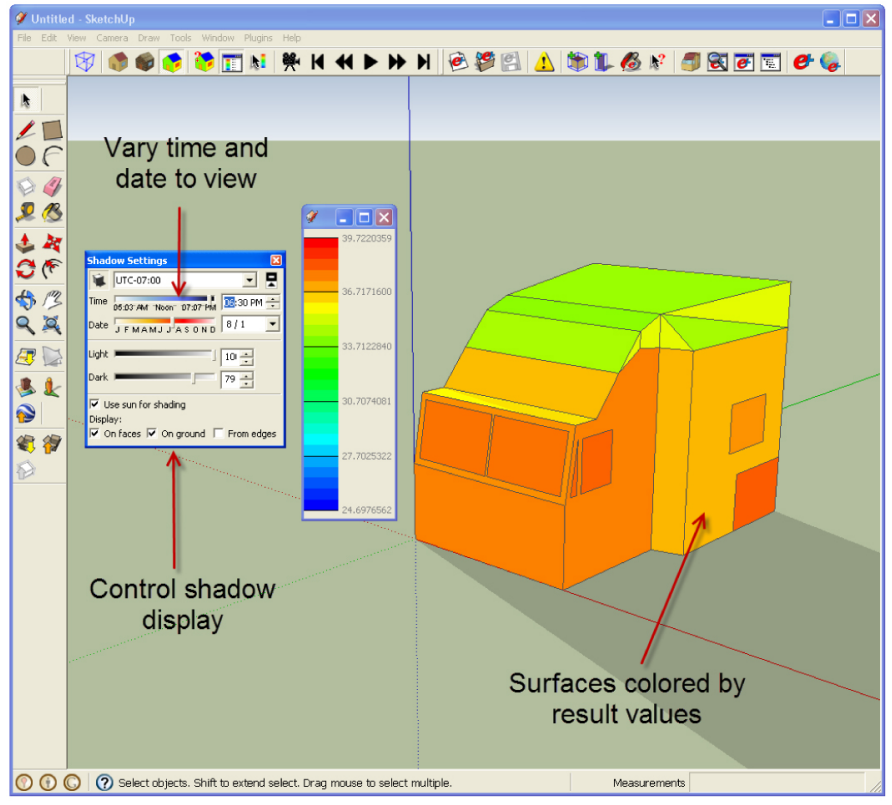

Figure 8. Example temperature distribution

\section{EXPERIMENTAL}

To validate the CoolCalc modeling approach, outdoor thermal soak testing of a Kenworth Truck Company T660 Class 8 sleeper cab truck was conducted at NREL's Vehicle Thermal Soak Test Facility (Figure 9). The truck was parked on level ground at an elevation of 5,853 feet, 39.7 degrees latitude, -105.2 degrees longitude, facing due south. The test vehicle was instrumented with 14 exterior and 28 interior ktype thermocouples. The thermocouples were calibrated using a Hart Scientific 7103 Micro-Bath for an overall uncertainty of less than $\pm 0.42^{\circ} \mathrm{C}$. The thermocouples were strategically positioned to accurately characterize the vehicle thermal distribution and collect validation data. Surface thermocouples were attached using an Omega thermally conductive epoxy. Radiation shields were used for air thermocouples to minimize errors due to direct solar radiation. A pyranometer was placed on the instrument panel of each vehicle to confirm sunrise times, and humidity measurements were taken. An IOTech LogBook 360 data acquisition system was used and located in the truck's toolbox. Data was collected every second and reduced to one minute averages over 24-hour periods. Solar radiation, wind speed, cloud coverage, relative humidity, and wind direction were also measured at NREL's Solar Radiation Research Laboratory, which is located near the test site.

Thermal soak tests were conducted from August through November 2009. The truck was exposed to daytime solar loads in an engine-off condition. An air exchange rate test was also conducted to help characterize thermal behavior. For this measurement, a small amount of sulfur hexafluoride $\left(\mathrm{SF}_{6}\right)$ was injected into the cab, and the concentration decay 
was monitored. Samples were taken every 5 minutes for 3 hours, and the air exchange rate was calculated from the $\mathrm{SF}_{6}$ concentration decay rate.



Figure 9. Test trucks at NREL's Vehicle Thermal Soak Test Facility

\section{VALIDATION}

To validate the CoolCalc simulation approach, a Kenworth T660 thermal model was developed. When available, information from Kenworth or testing was used to define the CoolCalc model parameters; otherwise, engineering estimates were made. Since CoolCalc does not use meshing, all surfaces must be planar. Therefore, the computer-aided design model provided by Kenworth was simplified. Figure 10 shows the model geometry compared to a photograph of the vehicle. The red lines indicated the domain boundary used for the model. This simplification process inherently requires some approximation; however, an effort was made to accurately represent the geometry.
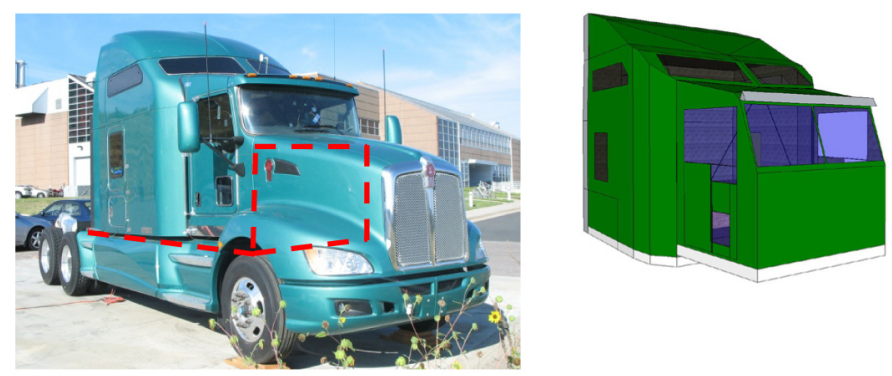

Figure 10. CoolCalc validation geometry

The vehicle was modeled with four air zones: Cab, Sleeper, Fairing, and Toolbox. The full EnergyPlus exterior radiation calculations were used; however, a simplified version of the interior radiation calculations was applied to increase geometry flexibility. The simple interior radiation model assumes that all beam radiation that passes through the windows falls on the floor of that zone and any reflected radiation is uniformly distributed on all interior surfaces. A previous case study was conducted that indicated that this results in increasing, but acceptable, differences during winter months due to the changing sun angle. The detailed surface convection algorithms were used that account for both natural and forced convection. The model time step was set to 1 minute intervals.

The glass properties for the cab and sleeper were obtained from Kenworth and its suppliers as were the materials and constructions for the vehicle walls. The overall resistivity of the walls was reduced to account for impacts of structural members and other likely disruptions to insulation in a real vehicle. The paint properties were not available and were estimated based on previous experience with vehicle paints.

Four internal masses were defined to represent interior objects. The first represents the seats in the cab. Specifications on vehicle seats were used to estimate the size, weight, and material properties. The next two internal masses represent the dashboard and the sleeper closets. Both were assumed to be polyurethane plastic, and the volume and surface area of each were estimated using truck geometry. Lastly, the two beds were modeled. Information on these was supplied by Kenworth. It was found that internal mass assumptions can have a significant impact on thermal results, and further work on internal mass modeling approaches is planned.

Since the chassis and hood would normally shade the underlying surfaces and were excluded from the solution domain, the solar load was removed from both surfaces. The firewall boundary condition was set to represent natural convection at $20 \%$ above ambient temperature. The exterior surfaces of the vehicle floor were exposed to wind at ambient temperature.

The measured air infiltration rate, as described in the experimental section, was applied to the cab and sleeper. Since this model does not include a fluid flow solution, the cross-mixing between zones had to be estimated. Incorporating a simplified means for estimating this is another possible improvement for the future.

\section{RESULTS}

The CoolCalc concept was validated by comparing the Kenworth T660 thermal model results with experimental temperature and onsite weather data. Figure 11 shows the weather data used for the three consecutive validation days. The data set has been normalized at the request of industry partners. This data set captures a range of conditions. The first day was overcast, cooler, and had low wind. The second two days were both clear, but had different wind and temperature characteristics. 


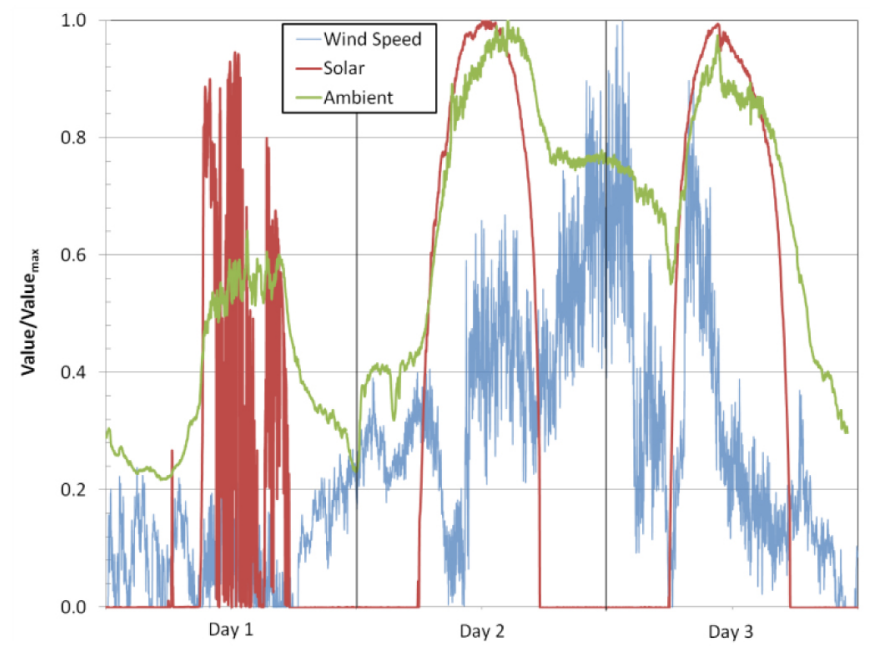

Figure 11. Normalized weather data for three validation days

The model solution time for the three-day simulation with 1minute intervals was $29.4 \mathrm{sec} \pm 0.1 \mathrm{sec}$ over three repeated runs using a Dell Intel Core 2 Duo 3.16 GHz CPU with 3.25 GB RAM desktop computer running the Windows XP operating system. Figure 12 and Figure 13 show a comparison between the model and the measured air temperatures. These graphs demonstrate good agreement between the model and the experimental data, both in the peak soak temperature and the overall trends. The experimental averages for the cab and sleeper are an average of six and eight distributed air temperatures, respectively.

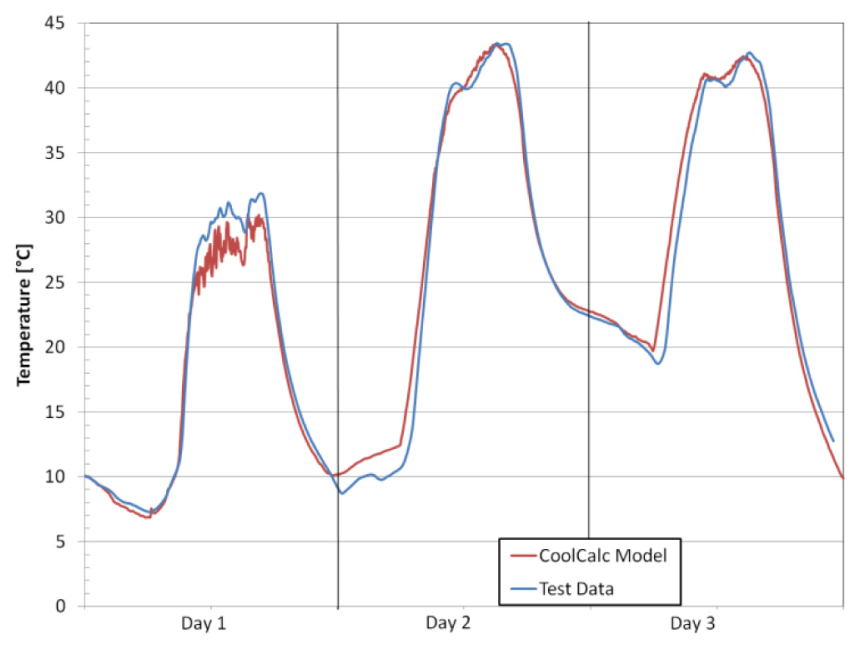

Figure 12. Cab average air temperature comparison

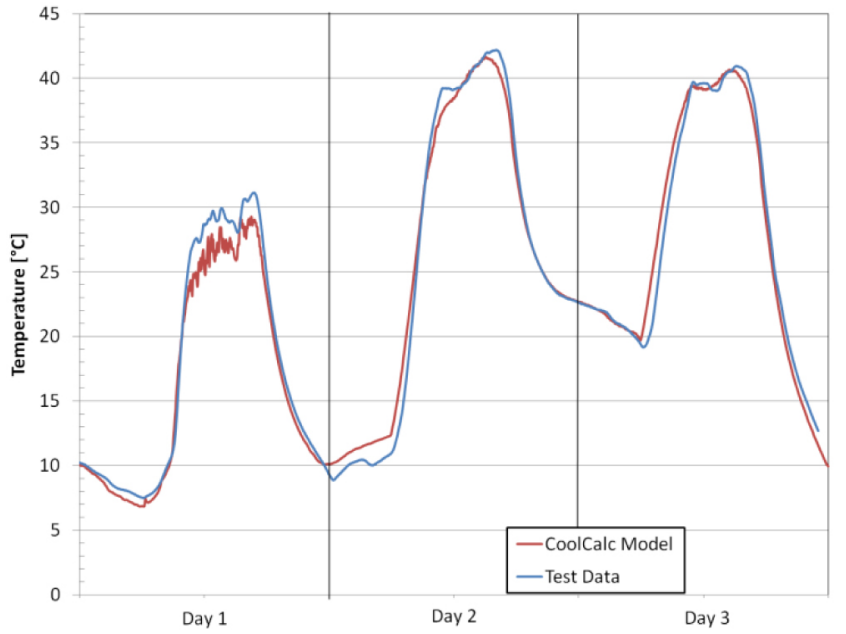

Figure 13. Sleeper average air temperature comparison

Figure 14 summarizes the temperature difference between the model and the test data for the time average (2-4 p.m.) sleeper and cab air temperatures. The maximum interior air temperatures occurred during this time interval and therefore represent peak soak conditions. For the sunny days, the temperature difference was less than $0.4^{\circ} \mathrm{C}$, which is less than the measurement uncertainty. On the cloudy day, the temperature difference between the data and the model was $2{ }^{\circ} \mathrm{C}$. While the average peak soak temperature for day two compares very closely with the experimental data, the differences with time can be seen in Figures 12 and 13 .

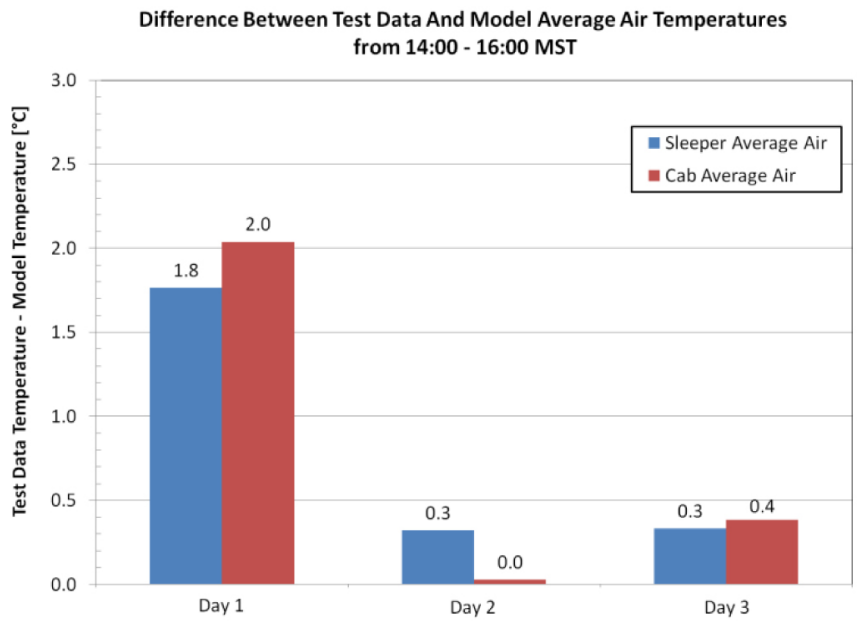

Figure 14. Comparison of test data and model, time average from 2-4 p.m.

The predicted exterior surface temperatures were also compared to the experimental results. Figure 15 shows the results for the driver and passenger sleeper side wall surface temperatures. Because the truck is facing south, the driverside surface temperatures rise in the morning, and then peak and decline as the sun passes over the vehicle. Likewise, the 
passenger-side surfaces rise in the afternoon, peak, and decline as the sun goes down. The higher frequency temporal variability seen in the afternoon temperatures on day one for both the experimental and the model results were caused by passing clouds. On day three, a larger error is seen between the model and the experimental data. Based on the high solar load and low wind speed, one possible cause for this could be that the natural convection portion of the heat transfer is underestimated in these conditions. However, further investigation would be needed to confirm this.

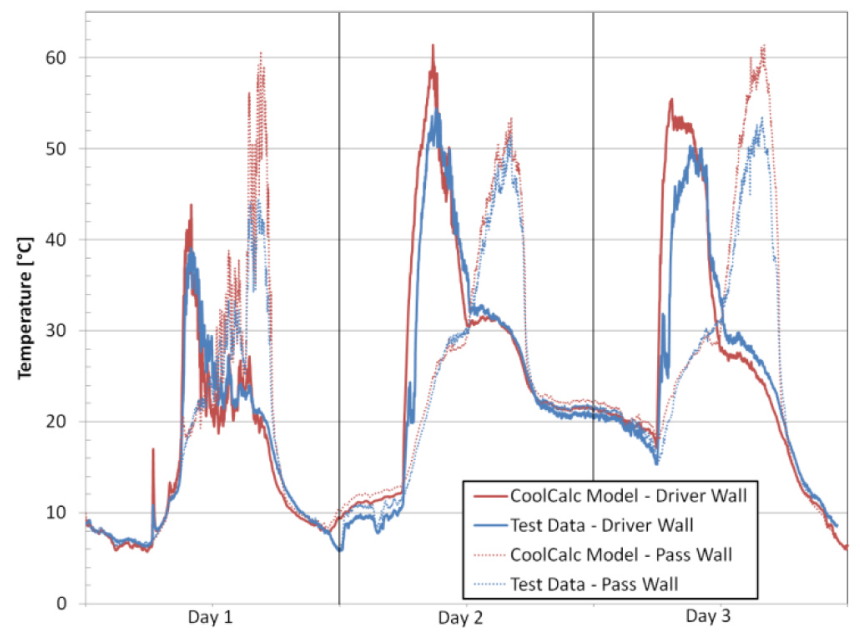

Figure 15. Sleeper driver and passenger exterior side walls

The concept validation results also show good agreement between the model and the experimental data for the other surfaces that were compared. Figure 16 shows the inside surface of the sleeper side windows. The model tends to overestimate the temperature and respond faster. EnergyPlus does not currently have thermal mass for windows; therefore, this behavior is expected. This could also account for the higher sensitivity to transients, such as passing clouds on Day1.

One of the larger differences between the model and the experimental results is the windshield, shown in Figure 17 and Figure 18. The temporal shift is more noticeable on the windshield exterior. This might also be caused by the lack of window thermal mass.

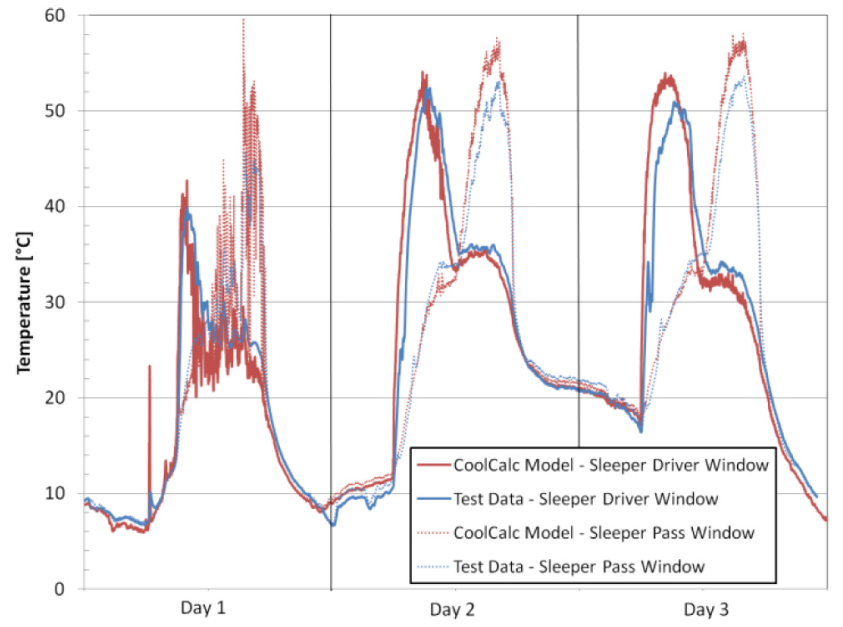

Figure 16. Comparison of sleeper interior side windows



Figure 17. Comparison of windshield exterior



Figure 18. Comparison of windshield interior 
These validation results demonstrate that the CoolCalc modeling tool can quickly and accurately estimate vehicle temperature distributions for air, surfaces, and glass. While inherent errors are expected when simplifying the geometry, this comparison to experimental results suggests that sufficient accuracy is achieved for early design and rapid trade-off analysis. Air conditioning tests were outside the scope of this first validation study. Matching the interior temperatures well during a thermal soak provides some confidence that the predicted air conditioning evaporator load is realistic. Previous work on simulation of military-type vehicle geometry indicated that the evaporator thermal load calculations were reasonable. Future work will validate load calculations in more detail. By modifying exterior convection coefficients as a function of speed and adding engine heat loads, down the road HVAC loads could be investigated in future work.

\section{SUMMARY/CONCLUSIONS}

To help develop solutions that reduce the 838 million gallons of fuel used by long-haul trucks annually for overnight idling in the United States, NREL's CoolCab project has developed an HVAC load estimation software tool called CoolCalc. A typical workflow for modeling the thermal behavior of a tractor trailer sleeper cab using CoolCalc was demonstrated. Vehicle geometry is first created using parametric and manual tools. The user navigates and modifies solver objects using the Object Browser as needed to define model parameters. Once the model is set up, the weather file is selected and the model is solved. Results can be displayed within the software environment or post-processed in detail using output files.

Detailed experimental testing of a Kenworth T660 sleeper cab truck was conducted at NREL to validate CoolCalc. A model of this vehicle was developed using information provided by Kenworth Truck Company, testing, and engineering assumptions. Comparison between the model and experimental results collected over three days shows good agreement both in trends and peak temperature values for a variety of weather conditions. The difference between experimental and model peak soak air temperatures, an average from 2-4 p.m., was less than or equal to $0.4^{\circ} \mathrm{C}$ for the two sunny days and $2^{\circ} \mathrm{C}$ for the cloudy day. Surface temperature comparisons show that the effect of solar position was captured accurately. Experimental testing for a second validation case study has been completed, and modeling is planned for the near future.

The ability of CoolCalc to quickly and accurately estimate vehicle thermal temperature distributions has been demonstrated. Further validation with other vehicles and weather conditions is being conducted. Methods and tools are currently being developed to link CoolCalc thermal load estimates to vehicle fuel use. The next step will be to apply
CoolCalc to study the impact of thermal load reduction technologies on idling and vehicle fuel use. This will fill an important role in the CoolCab project's suite of experimental and analytical tools that are being used to develop commercially viable idle reduction technologies in collaboration with industry partners.

\section{REFERENCES}

1. Stodolsky, F., Gaines, L., Vyas, A. Analysis of Technology Options to Reduce the Fuel Consumption of Idling Trucks. Argonne National Laboratory, ANL/ESD-43, June 2000.

2. Gaines, L., Vyas, A., Anderson, J. 2006, "Estimation of Fuel Use by Idling Commercial Trucks," 85th Annual Meeting of the Transportation Research Board, Washington, D.C., January 22-26, 2006, Paper No. 06-2567.

3. EnergyPlus Engineering Reference. http:// appsl.eere.energy.gov/buildings/energyplus/pdfs/ engineeringreference.pdf. October 11, 2010. Accessed on $[10 / 25 / 2010]$

\section{CONTACT INFORMATION}

Jason A. Lustbader

National Renewable Energy Laboratory

Jason.Lustbader@nrel.gov

303-275-4443

John P. Rugh

National Renewable Energy Laboratory

John.Rugh@nrel.gov

303-275-4413

\section{ACKNOWLEDGMENTS}

The authors would like to thank Lee Slezak and David Anderson, Technology Managers for DOE's Advanced Vehicle Technology Analysis and Evaluation for sponsoring this work.

A special thanks to John Duffy and Stan DeLizo of Kenworth Truck Company for their participation in and support of this work.

For their contributions to this project, thank you to the following NREL employees: Rob Farrington, Charlie King, Mike Lammert, Ken Proc, Bob Rehn, and Brent Griffith. Additionally, thank you to Michael Birdsong and Peter Ellis for your contributions to the program. 


\section{DEFINITIONS/ABBREVIATIONS}

\section{CAE}

computer-aided engineering

DOE

U.S. Department of Energy

HVAC

heating, ventilation, and air conditioning

\section{Kenworth}

Kenworth Truck Company

\section{NREL}

National Renewable Energy Laboratory

SF6

sulfur hexafluoride

The Engineering Meetings Board has approved this paper for publication. It has successfully completed SAE's peer review process under the supervision of the session organizer. This process requires a minimum of three (3) reviews by industry experts. ISSN 0148-7191
Positions and opinions advanced in this paper are those of the author(s) and not necessarily those of SAE. The author is solely responsible for the content of the paper.

SAE Customer Service:

Tel: 877-606-7323 (inside USA and Canada)

Tel: 724-776-4970 (outside USA)

Fax: 724-776-0790

Email: CustomerService@sae.org

SAE Web Address: http://www.sae.org

Printed in USA 